\title{
2D Stabilised analytic signal method in DC pole-pole potential data interpretation
}

\author{
Paras R PUjari* and Rambhatla G SASTRY** \\ *Scientist, National Environmental Engineering Research Institute (NEERI), Nehru Marg, Nagpur 440 020, India \\ e-mail: prpujari@nagpur.dot.net.in \\ ** Associate Professor, Department of Earth Sciences, IIT, Roorkee 247 667, India \\ e-mail:rgss1fes@rurkiu.ernet.in_rgssastry@yahoo.com
}

Using analytic signal method, interpretation of pole-pole secondary electric potentials due to $2 \mathrm{D}$ conductive/resistive prisms is presented. The estimated parameters are the location, lateral extent or width and depth to top surface of the prism. Forward modelling is attempted by 2D-Finite Difference method. The proposed stabilised analytic signal algorithm (RES2AS) uses Tikhonov's regularization scheme and FFT routines. The algorithm is tested on three theoretical examples and field data from the campus of Roorkee University. The stability of RES2AS is also tested on synthetic error prone secondary pole-pole potential data.

\section{Introduction}

Analytical signal method (Nabighian 1972; Roest et al 1992) or its variants (Rao, Babu and Narayan 1981; Sundarrajan and Srinivas 1996; Sundarrajan, Rao and Sunitha 1998) have been proposed in the past for interpretation of potential field anomalies and S.P anomalies. However, its application to DC electrical signals is not yet reported. The success of analytic signal method (Nabighian 1972, 1984; Roest et al 1992) and the formal analogy between magnetostatics and electrostatics (Eskola 1992; Quick 1974) allow its use (Pujari 1998) in the analysis of DC pole-pole secondary electric potential data caused by $2-\mathrm{D}$ conductive/resistive closed bodies of rectangular cross-section. Here, the similarity of secondary potential, $V_{s}$ with the residual magnetic field anomaly interpretation is invoked.

The complex analytic signal computation depends on stable numerical derivatives of secondary electric potentials. So, Tikhonov's regularization is incorporated in the designed analytic algorithm. Theoretical modelling is carried out on an isolated 2D conductive/resistive body of rectangular cross-section enclosed within resistive/conductive host medium.

\section{Similarity in governing equations of magnetostatics and electrostatics}

A fundamental feature of Maxwell's equations is their symmetry in relation to the electric and magnetic fields.

In the case of magnetostatics, the magnetic scalar potential, $u^{*}$ obeys the governing equation

$$
\nabla^{2} u^{*}=-\sigma^{*}
$$

in an anomalous region, where the volume density of magnetic poles is $\sigma^{*}$.

In a source-free region, it assumes

$$
\nabla^{2} u^{*}=0 .
$$

Further, across a boundary separating two media with different magnetic permeabilities, $\mu_{1}$ and $\mu_{2}$, the scalar magnetic potential satisfies the following two boundary conditions

$$
u_{1}^{*}=u_{2}^{*}
$$

Keywords. Analytic signal; regularisation; secondary electric potentials. 


$$
\mu_{1} \frac{\partial u_{1}^{*}}{\partial n}=\mu_{2} \frac{\partial u_{1}^{*}}{\partial n}
$$

where $\frac{\partial}{\partial n}$ refers to derivative normal to the interface.

In the case of electrostatics, the governing equation for electric potential, $u$, in a medium where there is charge injection, $I$ is given by

$$
\nabla^{2} u=-I \text {. }
$$

In a source-free region, the electric potential satisfies

$$
\nabla^{2} u=0
$$

The electric potential, $u$ also satisfies the following two boundary conditions across a physical interface separating two media with resistivities $\rho_{1}$ and $\rho_{2}$

$$
\begin{aligned}
u_{1} & =u_{2}, \\
\frac{1}{\rho_{1}} \frac{\partial u_{1}}{\partial n} & =\frac{1}{\rho_{2}} \frac{\partial u_{2}}{\partial n}
\end{aligned}
$$

where $\hat{n}$ refers to unit normal to the interface.

Thus, the governing equations along with boundary conditions for static electrical and magnetic fields are similar.

\section{Analogy of secondary electric potential and residual magnetic anomalies}

In a typical DC resistivity profiling, the measured total potential comprises of primary and secondary components, the former one relates to the response of host medium and the latter to that of the target in question. In all resistivity imaging problems, the emphasis is laid on secondary potential estimation for further analysis. In the magnetic method, the processed anomalies are subjected to regionalresidual separation to separate out residual anomalies, which are interpreted for geological object(s) in question.

Thus, the secondary electric potential is equivalent to residual magnetic anomaly. However, this analogy is restricted to closed bodies in view of charge accumulation concepts ( $\mathrm{Li}$ and Oldenburg 1991) due to external current source(s) and $\operatorname{sink}(\mathrm{s})$ pairs and it fails for target(s) with open electrical interface(s) due to non-uniform polarisation.

\section{Analytic Signal (AS) and related parameters}

Following Nabighian (1972), the 2-D Analytic Signal (AS), $A(x, z)$ of the secondary pole-pole potential $V_{s}(x, z)$ can be defined as

$$
A(x, z)=\frac{\partial V_{s}^{\prime}}{\partial x}+\hat{j} \frac{\partial V_{s}^{\prime}}{\partial z}
$$

where $\hat{J}=\sqrt{-1}$.

The amplitude of the analytic signal (AS) is given by

$$
|A(x, z)|=\sqrt{\left(\frac{\partial V_{s}^{\prime}}{\partial x}\right)^{2}+\left(\frac{\partial V_{s}^{\prime}}{\partial z}\right)^{2}}
$$

where $V_{s}^{\prime}=\frac{\partial V_{s}}{\partial x}$.

The real and imaginary parts of R.H.S of (5) constitute a Hilbert transform pair (Nabighian 1972).

Nabighian (1972) has identified the following properties of complex Analytic Signal of magnetic anomaly due to a 2 -d body of arbitrary cross-section, approximated by an $n$-sided polygon.

- The amplitude of analytic signal, AAS is a symmetrical function maximising exactly over the top of each corner of the 2-d body.

- The complex analytic signal, AS has simple poles at each corner of the arbitrary shaped 2-d body and conversely, $1 / A(x, z)$ has zeros at those corners. So, the real part of the inverse of AS is zero at the body corners.

Thus, the AAS can be used for computing the depth to top of the body and the poles of AS fix the lateral edges of the body.

The various AS parameters relevant for interpretation are

- Amplitude of Analytic Signal (AAS).

- Amplitude of the Real part of $1 / \mathrm{A}(x, z)$ i.e., (RIAS).

- Real component of the Analytic Signal (RAS).

- Imaginary component of the Analytic Signal (IAS).

The RAS and IAS are used for determining the position of the centre of the body, whereas the AAS is used for defining the depth to top of the body. The RIAS, which gives the zeros of the inverse of AS is used to find the lateral coordinates or lateral extent of the body.

For depth determination, only a simple halfwidth depth rule (Nabighians 1972) has been used.

\section{Computation of Stable Analytic Signal}

In view of numerical derivative computations being unstable (Tikhonov \& Arsenin 1977), analytic sig- 
nal computation is an ill-posed problem. So, our spectral algorithm (RES2AS) uses both regularisation strategy (Tikhonov \& Arsenin 1977) and FFT routines (Starostenko \& Sastry 1979) for computing a stable analytic signal from secondary potential data.

Computation of $n$th order derivative of a given arbitrary function $u(x)$ is governed by Volterra type Integral equation (Tikhonov \& Arsenin 1977) of first kind of convolution type given by

$$
\int_{0}^{t} \frac{1}{(n-1) !}(t-\xi)^{(n-1)} z(\xi) d \xi=u(t)
$$

which is ill-posed.

In operator form, (7) can be written as

$$
A z=u
$$

where $z \in Z, u \in U$ with $Z$ and $U$ as Hilbert spaces. by

In Fourier domain, the spectrum of $z(t)$ is given

$$
\tilde{Z}(\omega)=\frac{\tilde{U}(\omega)}{\tilde{K}(\omega)}
$$

where the Fourier transform pairs are

$$
\begin{aligned}
z(t) & \Leftrightarrow \tilde{Z}(\omega) \\
k(t) & \Leftrightarrow \tilde{K}(\omega) \\
u(t) & \Leftrightarrow \tilde{U}(\omega)
\end{aligned}
$$

and $\omega$, is the spatial frequency and $k(t)$, the kernel of (1).

However, in view of the ill-posedness of (8), Inverse Fourier Transform, IFFT of (7) does not exist.

The regularised solution as per Tikhonov and Arsenin (1977) is given by

$$
Z_{\alpha}(t)=\operatorname{IFFT}\left(\frac{\tilde{u}(\omega)}{\tilde{k}(\omega)} f(\omega, \alpha)\right) .
$$
be

The regularisation operator, $f(\omega, \alpha)$ is chosen to

$$
f(\omega, \alpha)=\frac{1}{1+\alpha M(\omega)},
$$

where $M(\omega)$ is a smoothing function.

As per the theory of regularisation (Tikhonov \& Arsenin 1977), one can choose $M(\omega)$ to be of the form

$$
M(\omega)=\omega^{2 p}
$$

where $p$, the order of regularisation, controls the degree of smoothness intended for the solution. For our numerical experiments, $p=2$.
Thus, 2-D Stabilised Analytic Signal algorithm, RES2AS is designed.

\section{Interpretation procedure}

The sequence of interpretation steps is outlined in a flowchart (figure 1). They entail two basic tasks.

- Computation of secondary potential (pole-pole).

- Computation of stable AS and its associated terms, which are used for the semi-quantitative interpretation.

\subsection{Computation of secondary pole-pole potential}

In numerical experiments, secondary pole-pole electric potential distribution at air-earth interface is generated by subtracting the host medium effect from the total measured potential due to combined effect of host and target body for a fixed current source and sink configuration using a finitedifference (Mufti 1976) scheme.

In field survey, for a fixed source(s) and $\operatorname{sink}(\mathrm{s})$ configuration, the host medium response at receiver electrodes is estimated by conducting the experiment outside the influence of the target body. Then, in the vicinity of the body, a staggered set of receiver electrodes are considered where the total potential is measured for similar arrangement of source(s) and receiver(s). A difference of these two responses for each receiver electrode position in a pole-pole set up provides the desired secondary electric pole-pole potential, for further analysis by RES2AS.The included case study employs this methodology.

\section{Numerical experiments and results}

The input data to RES2AS could either be error-free or error-prone DC pole-pole secondary potential data due to conductive/resistive target(s) located within resistive/ conductive halfspace respectively.

A 2-D body of size $6 \mathrm{~m} \times 6 \mathrm{~m}$, with a square cross-section constitutes the model for theoretical studies. Figure 2 is a schematic diagram explaining the emplacement of the body in the linear portion of the FDM mesh $(51 \times 101$ nodes $)$ along with the source positions $S_{0}$, and $S_{1}$ at 33 , and 28 node positions respectively. The linear portion of the mesh extends over 64 nodes in the $X$-direction.

The target details inclusive of physical property distribution and source strength are provided in table 1, whereas table 2 contains the geometric details of the considered models. 
Obtain secondary potential distribution, $V^{F}$ at air earth interface from pole pole field data.

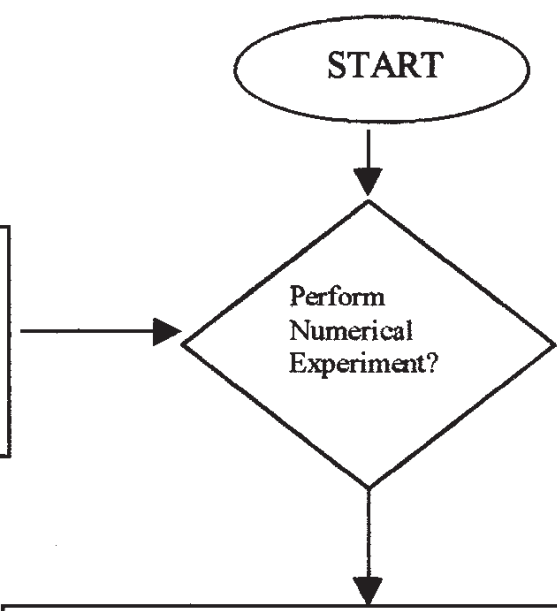

Compute total response (Total potential, $\mathrm{V}_{\mathrm{T}}(\mathrm{x})$ ) at air earth interface due to a chosen conductivity model within resistive half-space to single current source (pole-pole configuration) as well as due to resistive half space(potential $\mathrm{V}_{\mathrm{H}}(\mathrm{x})$ ) by FDM algorithm of RES2AS.

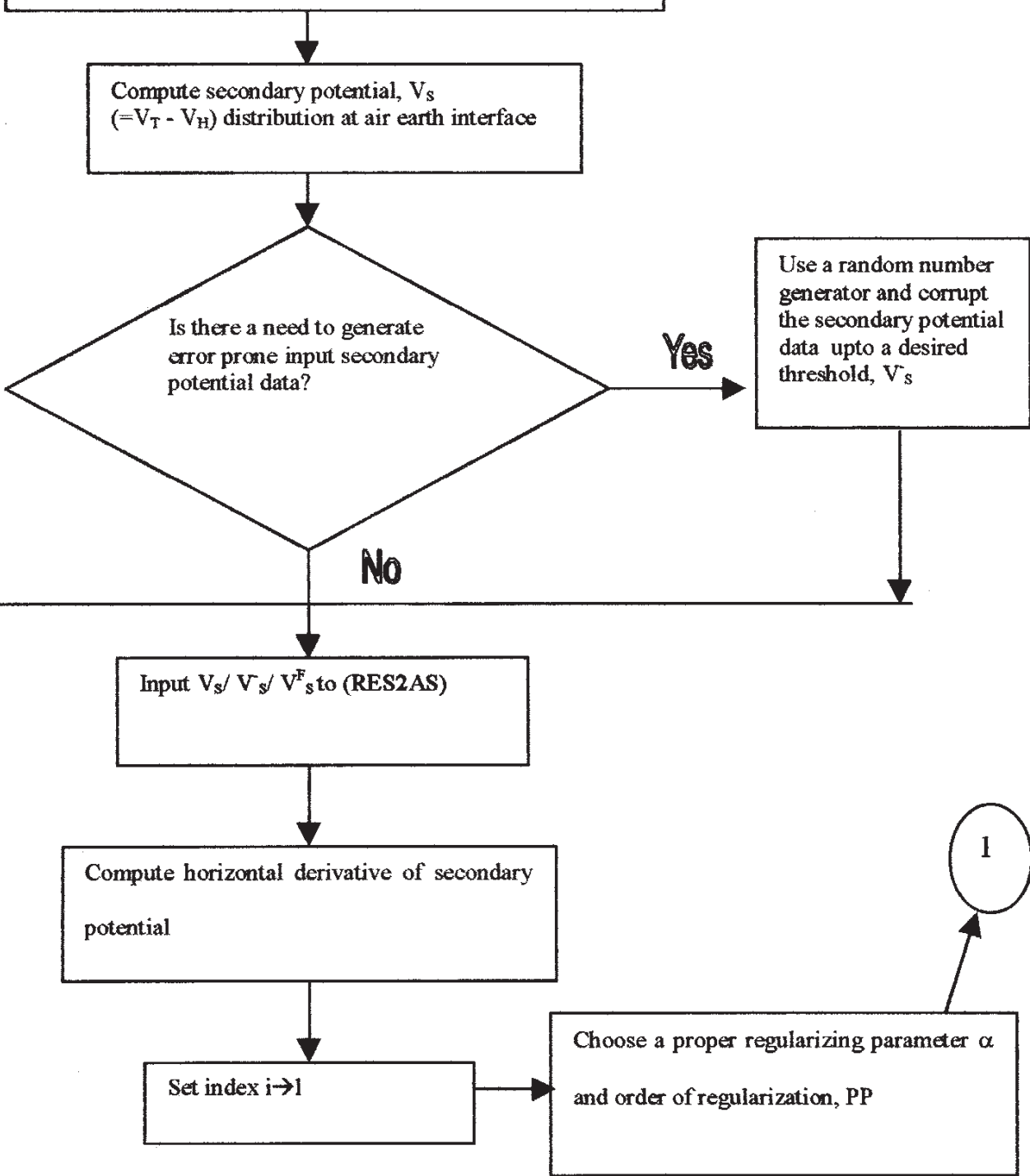

Figure 1. Flow chart giving the sequence of interpretation steps of RES2AS algorithm. 


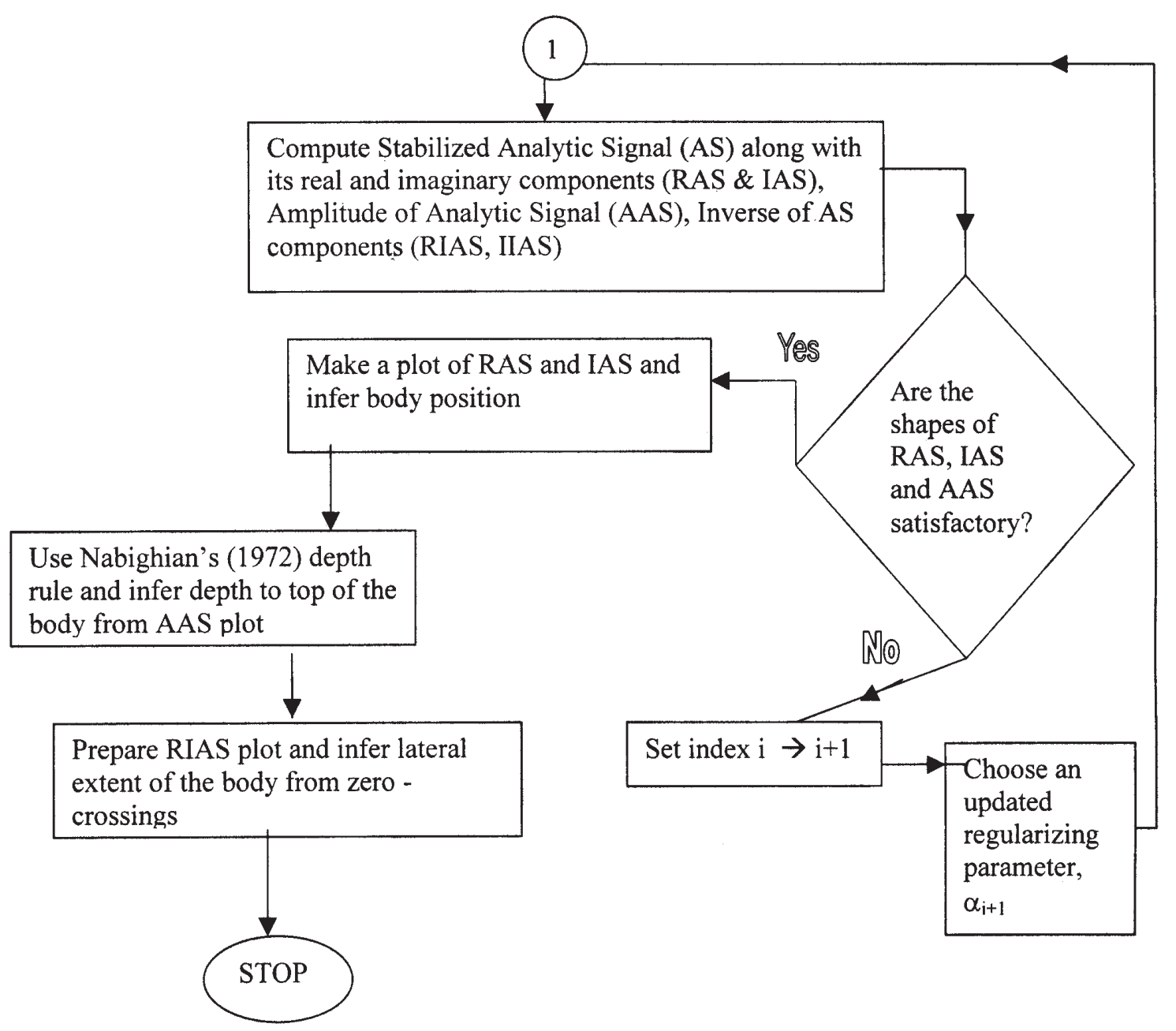

Figure 1. (Continued)

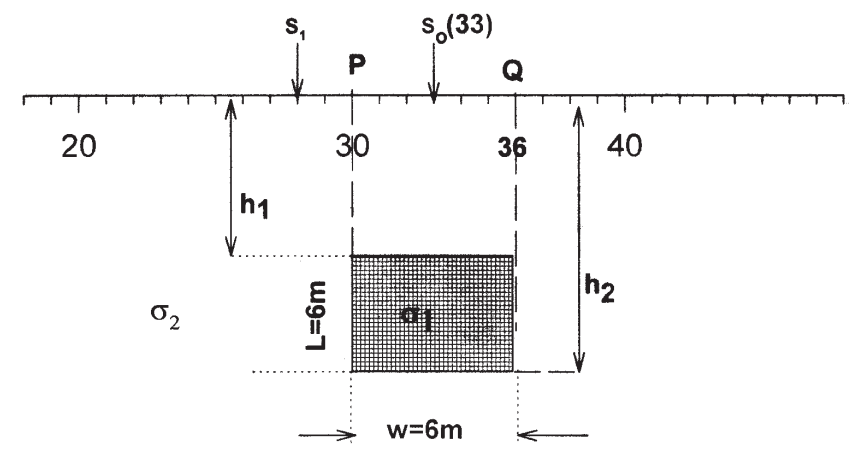

Figure 2. Schematic section of the linear portion of the finite-difference mesh containing the anomalous body (Model 1) with current source positions $S_{0}$ and $S_{1}$ at nodes 33 and 28 respectively. $\sigma_{1}$ and $\sigma_{2}$ are conductivities in $\mathrm{s} / \mathrm{m}$ of inhomogeneity and background half space respectively. The meaning of the rest of the parameters is selfevident.

The three versions of models are referred to as Model 1.1, Model 1.2 and Model 1.3 respectively
Table 1. Numerical values for current source strength, conductivities of the inhomogeneity and background half-space.

\begin{tabular}{lccc}
\hline \multicolumn{1}{c}{ Models } & $\begin{array}{c}\text { Current } \\
\text { strength } \\
(\mathrm{A} / \mathrm{m})\end{array}$ & $\begin{array}{c}\text { Conductivity of } \\
\text { inhomogeneity } \\
(\mathrm{S} / \mathrm{m})\end{array}$ & $\begin{array}{c}\text { Conductivity } \\
\text { of half-space } \\
(\mathrm{S} / \mathrm{m})\end{array}$ \\
\hline $\begin{array}{l}1.1,1.2,1.3 \\
\text { (Conductive) }\end{array}$ & 1 & 0.1 & 0.01 \\
$\begin{array}{l}1.1 \\
\text { (Resistive) }\end{array}$ & 1 & 0.01 & 0.1 \\
\hline
\end{tabular}

(refer table 2). Interpretation as per the earlier outlined procedure (figure 1) is implemented.

\subsection{Model 1.1}

\section{Case I: Conductive body}

For illustration sake, the position location of the centre of the target is highlighted only for the model 1.1. 
Table 2. Geometric details of Model 1. (Schematic section is given in figure 2).

\begin{tabular}{rccccc}
\hline Prism & $\begin{array}{c}\text { Lateral } \\
\text { extent } \\
(\mathrm{P}, \mathrm{Q})\end{array}$ & $\begin{array}{c}\text { Depth to } \\
\text { top }(\mathrm{m}) \\
\mathrm{h} 1\end{array}$ & $\begin{array}{c}\text { Depth to } \\
\text { bottom }(\mathrm{m}) \\
\mathrm{h} 2\end{array}$ & $\begin{array}{c}\text { Depth to } \\
\text { center }(\mathrm{m}) \\
\mathrm{H}\end{array}$ & $\begin{array}{c}\text { Position of } \\
\text { body } \\
\text { center }\end{array}$ \\
\hline Model 1.1 & $(30,36)$ & 3 & 9 & 6 & 33 \\
Model 1.2 & $(30,36)$ & 2 & 8 & 5 & 33 \\
Model 1.3 & $(30,36)$ & 5 & 11 & 8 & 33 \\
\hline
\end{tabular}

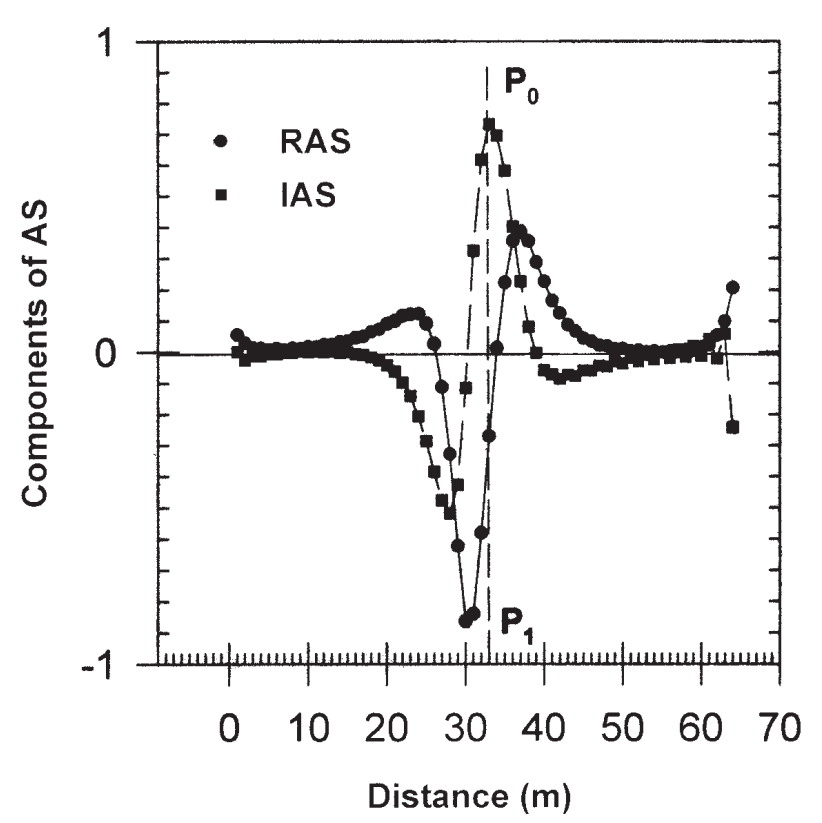

Figure 3. Real and imaginary component plots of analytic signal (RAS and IAS) for Model 1.1 (refer tables 1 and 2), when current source is offset from body axis, $P_{0} P_{1}\left(S_{1}\right.$ in figure 2).

\section{Determination of position of the centre of the body}

Two different source positions, $S_{1}$ and $S_{0}$ (refer figure 2) are considered. For $S_{1}$, the plots of RAS and IAS (figure 3 ) are neither symmetric nor antisymmetric about the vertical axis $\left(P_{0} P_{1}\right)$ through the body centre, whereas for $S_{0}$ (refer figure 4 ) exactly above body centre, they are perfectly symmetric and anti-symmetric respectively.

\section{Determination of depth to top of the body}

The half-width depth rule of Nabighian (1972) determines the depth to top (figure 5) of the body. The depth estimate is included in table 3 .

\section{Determination of the lateral extent of the body}

Figure 6 represents the plot of RIAS. The inferred body edges from the zero crossings are presented in table 3 .

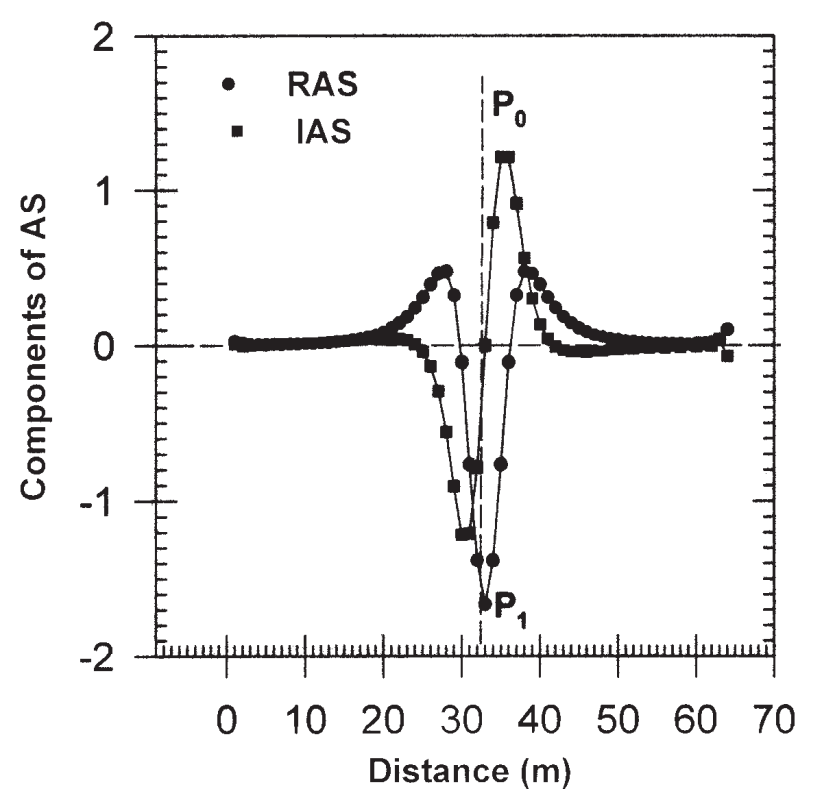

Figure 4. Real and imaginary component plots of analytic signal (RAS and IAS) for Model 1.1 (refer tables 1 and 2), when current source is at projection of body axis, $P_{0} P_{1}\left(S_{0}\right.$ in figure 2).

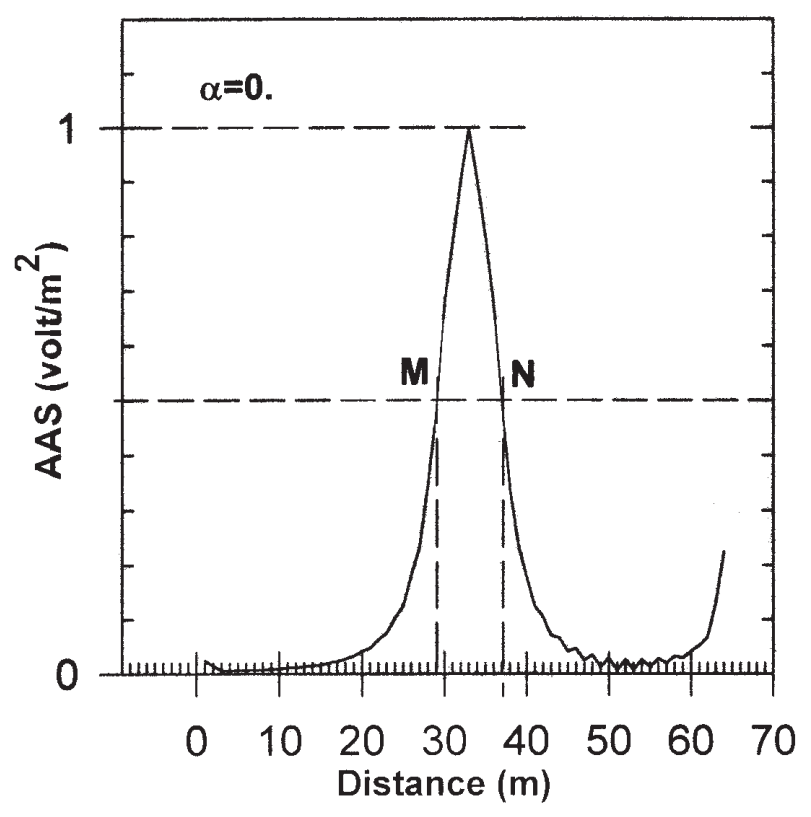

Figure 5. Amplitude of analytic signal (AAS) plot for Model 1.1 and computation of depth to top surface of the body by Nabighian's depth rule. MN is full width. MN/2 is the estimated depth to top (refer table. 3 ). Source is at $S_{0}$. 
Table 3. Results of numerical experiments for square shaped 2-D body of size $6 \mathrm{~m} \times 6 \mathrm{~m}$.

\begin{tabular}{|c|c|c|c|c|}
\hline Model & $\begin{array}{l}\text { Actual } \\
\text { depth } \\
(\mathrm{m})\end{array}$ & $\begin{array}{l}\text { Inferred } \\
\text { depth } \\
(\mathrm{m})\end{array}$ & $\begin{array}{c}\text { Actual } \\
\text { lateral } \\
\text { extent } \\
\text { (node-wise) }\end{array}$ & $\begin{array}{c}\text { Inferred } \\
\text { lateral } \\
\text { extent } \\
\text { (node-wise) }\end{array}$ \\
\hline $\begin{array}{l}1.1 \\
\text { (Conductive } \\
\text { case) }\end{array}$ & 3 & 4 & $(30,36)$ & $(30,36)$ \\
\hline $\begin{array}{l}1.1 \\
\text { (Noisy data) }\end{array}$ & 3 & 5.5 & $(30,36)$ & $(28,38)$ \\
\hline $\begin{array}{l}1.1 \\
\text { (Resistive } \\
\text { case) }\end{array}$ & 3 & 4 & $(30,36)$ & $(30,36)$ \\
\hline $\begin{array}{l}1.2 \\
\text { (Conductive } \\
\text { case) }\end{array}$ & 2 & 2 & $(30,36)$ & $(30,37)$ \\
\hline 1.3 & 5 & 7 & $(30,36)$ & $(28,37)$ \\
\hline
\end{tabular}

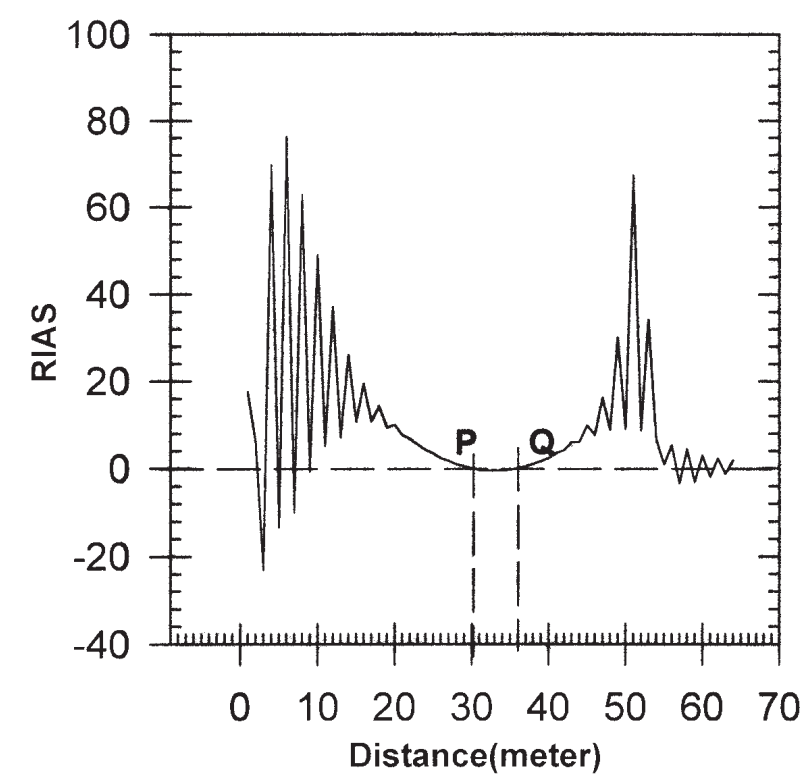

Figure 6. Real component of complex inverse analytic signal (RIAS) plot for Model 1.1 (refer tables 1 and 2). Its zero crossings at $P$ and $Q$ identify the lateral edges of the body (refer table. 3). Source is at $S_{0}$.

\section{Stability of the algorithm}

The stability of RES2AS is checked by adding $5 \%$ random Gaussian noise to the secondary potential due to Model 1.1. Figure 7 is for unregularised case and figure 8 is for regularised. In case of regularisation $(\alpha=7$ ), the AAS (figure 8) behaves well and has been used for depth estimation. The RIAS (figure 9) identifies the body edges at nodes $(29,37)$ which are not far from the actual ones. Table 3 enlists the results.

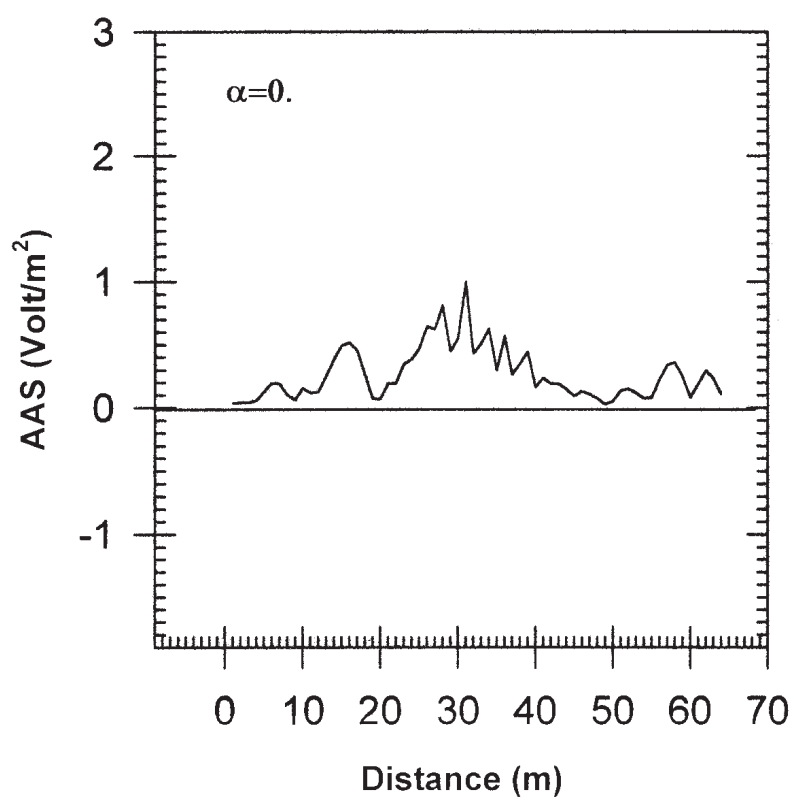

Figure 7. Amplitude of analytic signal (AAS) plot for Model 1.1 (conductive case) for error-prone input pole-pole potential data. A 5\% Gaussian noise is included in the input data. No regularisation is incorporated.

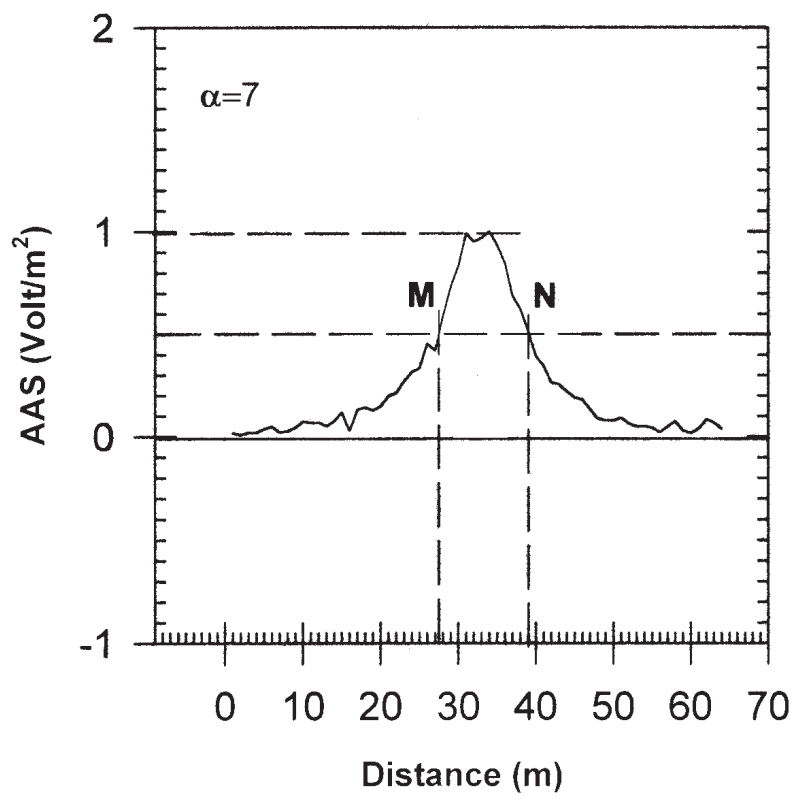

Figure 8. Regularised amplitude of analytic signal (AAS) plot (regularisation parameter, $\alpha=7.0$ ). A $5 \%$ Gaussian noise is included in the input data (refer figure 7 ).

\section{Case 2: Resistive body}

The geometry of the body remains the same as that of Model 1.1 (refer table 2) and table 1 contains physical property distribution and source details. Two source positions are considered i.e., $S_{1}$ and $S_{0}$ (refer figure 2). Body centre is located correctly for source $S_{0}$ (figure 10) in comparison to that of $S_{1}$ (figure 11). Depth and lateral extent of the body 


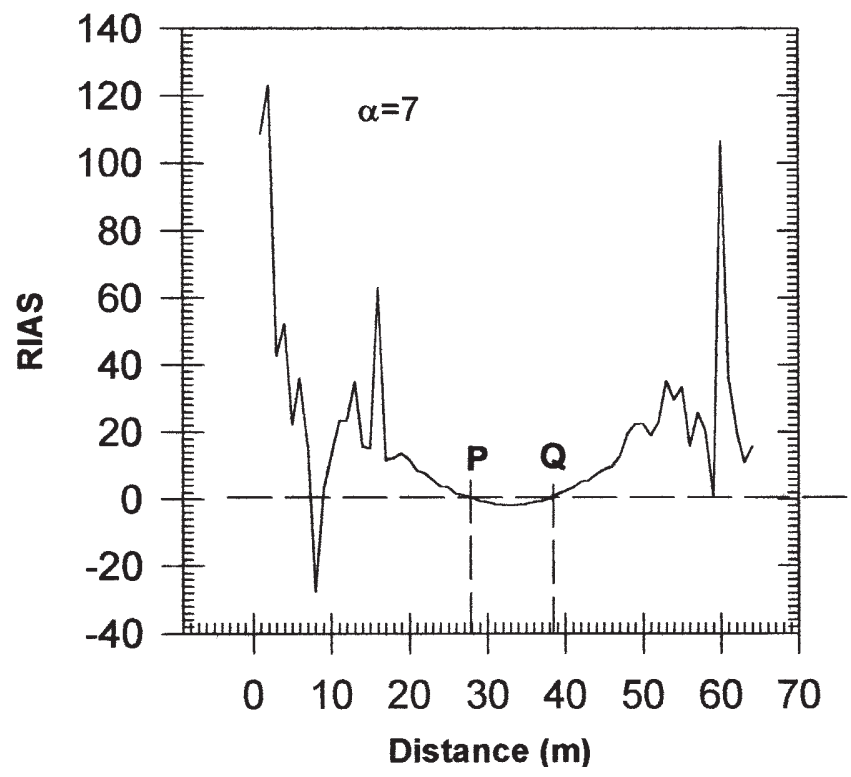

Figure 9. Regularised $(\alpha=7.0)$ real component of complex inverse analytic signal (RIAS) plot for error-prone input pole-pole potential data (5\% Gaussian noise) due to Model 1.1 (conductive case).

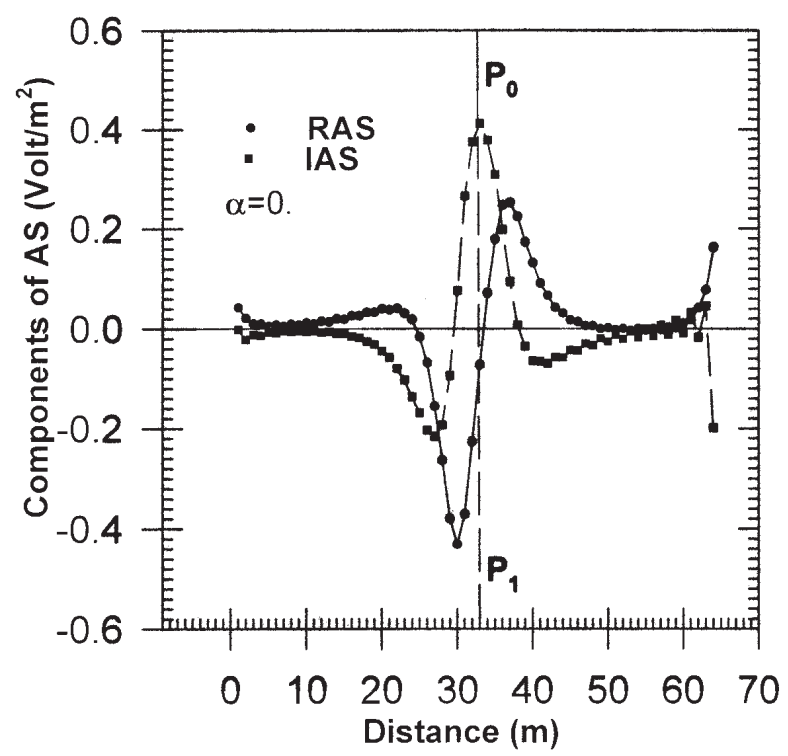

Figure 10. Real and imaginary component plots of analytic signal (RAS and IAS) for Model 1.1 (resistive case), when current source is offset from body axis, $P_{0} P_{1}\left(S_{1}\right.$ in figure 2$)$. Source is at $S_{1}$ (refer figure 2).

determined from figures 12 and 13 respectively are presented in table 3.

\subsection{Model 1.2 and Model 1.3}

The earlier exercise is repeated for Model 1.2 and Model 1.3. For the sake of brevity, the plots of RAS and IAS are not reproduced here. The depth to top and lateral extent of the body are determined from AAS and RIAS plots (figures 14 and 15) for

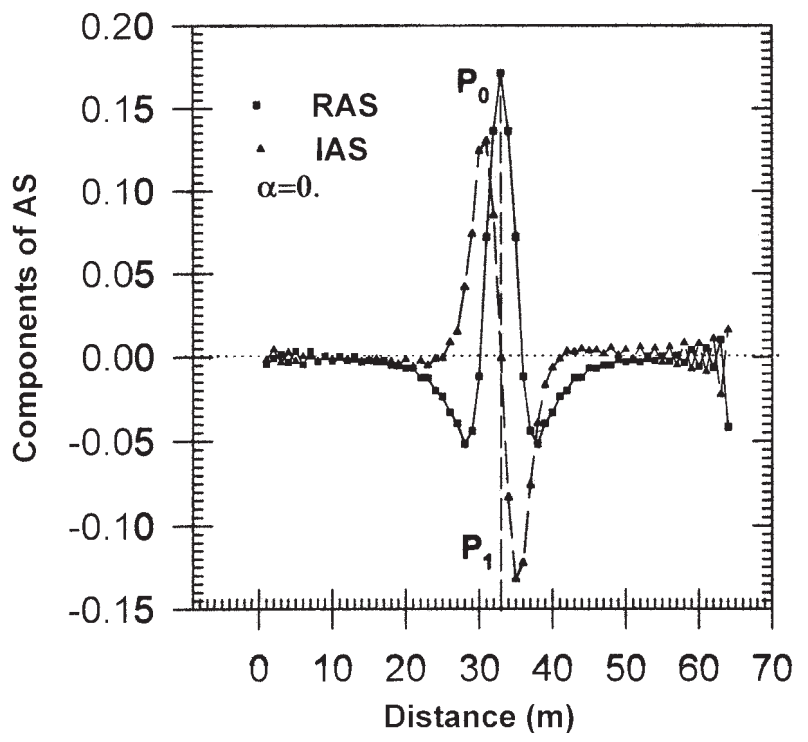

Figure 11. Real and imaginary component plots of analytic signal (RAS and IAS) for Model 1.1 (resistive case) (refer tables 1 and 2), when current source is at $S_{0}$, projection of body axis, $P_{0} P_{1}$ (refer figure 2 ).

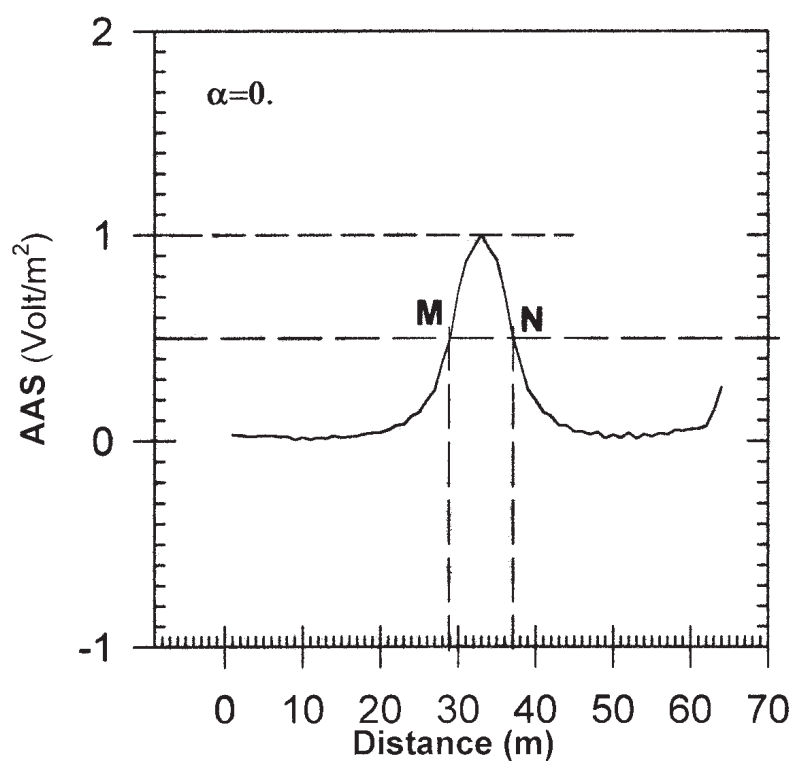

Figure 12. Amplitude of analytic signal (AAS) plot for Model 1.1 (resistive case) and computation of depth to top surface of the body by Nabighian's depth rule. MN is full width. MN/2 is the estimated depth to top (refer table 3 ). Source is at $S_{0}$.

Model 1.2 and (figures 16 and 17) for Model 1.3 are included in table 3 .

\section{Field example}

The objective is to locate and determine lateral extent of an iron pipe of small diameter, which drains waste water from our institute's swimming pool located on the campus (Lat. 29052' 11.9", Long. $\left.77053^{\prime} 37.1^{\prime \prime} \mathrm{E}\right)$. 


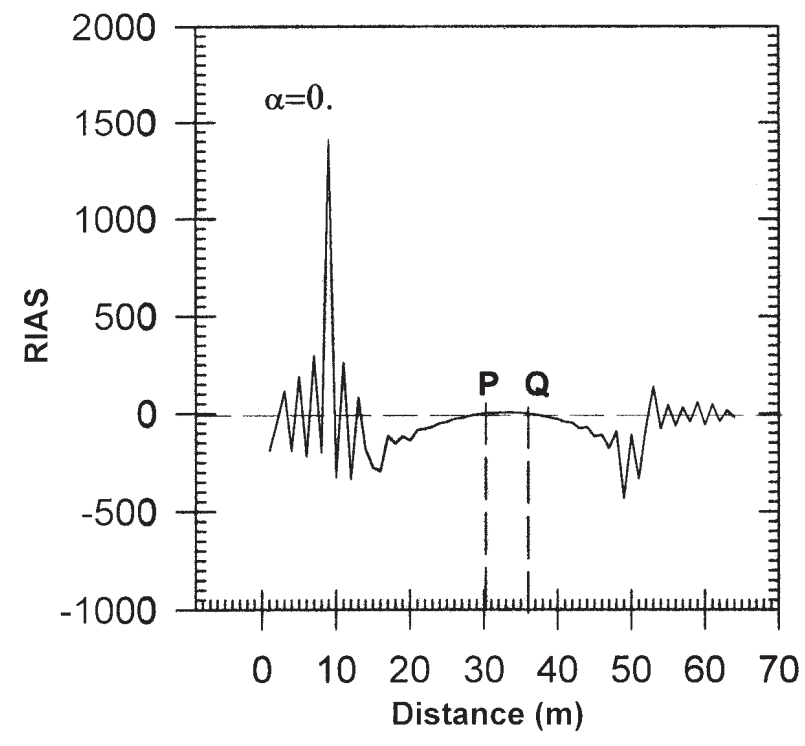

Figure 13. Real component of complex inverse analytic signal (RIAS) plot for Model 1.1 (resistive case) (refer tables 1 and 2). Its zero crossings at $P$ and $Q$ identify the lateral edges of the body (refer table 3 ). Source is at $S_{0}$.

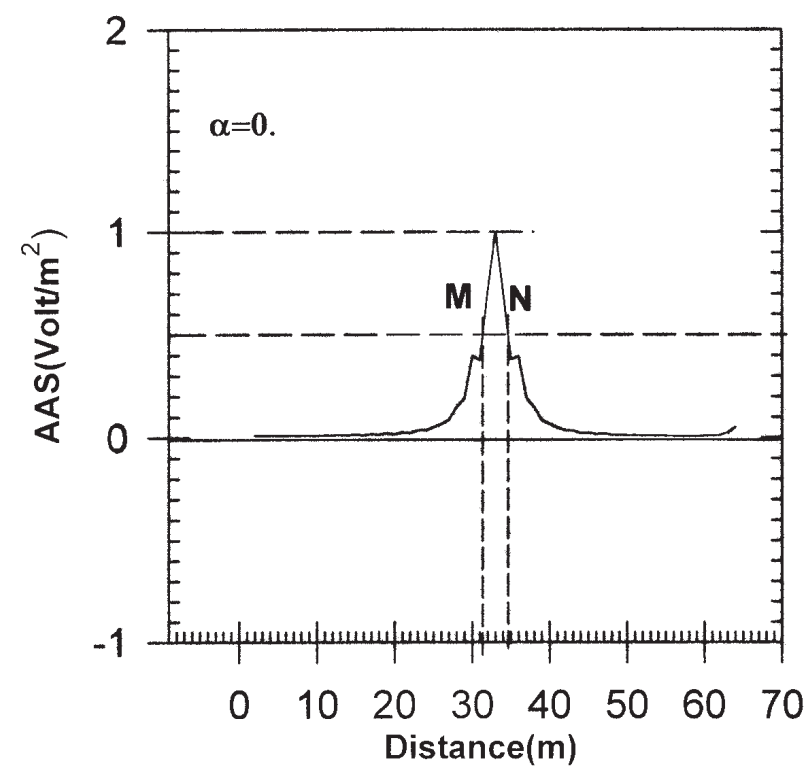

Figure 14. Amplitude of analytic signal (AAS) plot for Model 1.2 and computation of depth to top surface of the body by Nabighian's depth rule. MN is full width. MN/2 is the estimated depth to top (refer table 3 ). Source is at $S_{0}$.

The geometric details of the drain pipe as gathered from the institute records are included in table 4. A separate VES is performed in the region to assess the background conductivity of the soil cover within which the pipe is embedded. Figure 18(a) provides a schematic field layout in plan and figure 18(b) bears the depth-section including the current pole positions. Pole-Pole electrode configuration is used here. The separation between two adjacent stations (say $1 \mathrm{~N}$ and $2 \mathrm{~N}$ ) is $0.1524 \mathrm{~m}$.

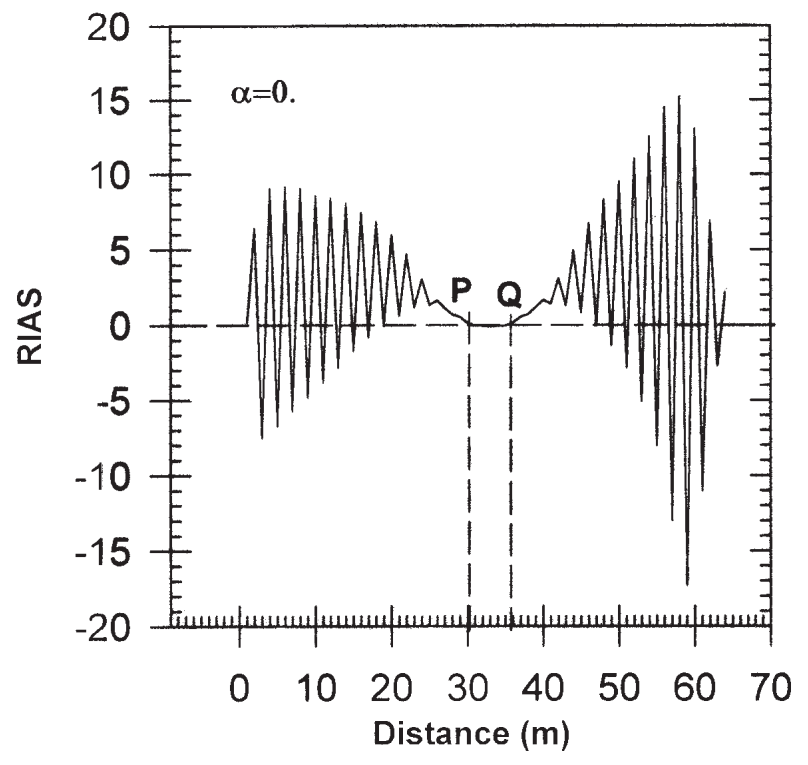

Figure 15. Real amplitude of complex inverse analytic signal (RIAS) plot for Model 1.2 (refer tables 1 and 2). Its zero crossings at $P$ and $Q$ identify the lateral edges of the body (refer table 3). Source is at $S_{0}$.

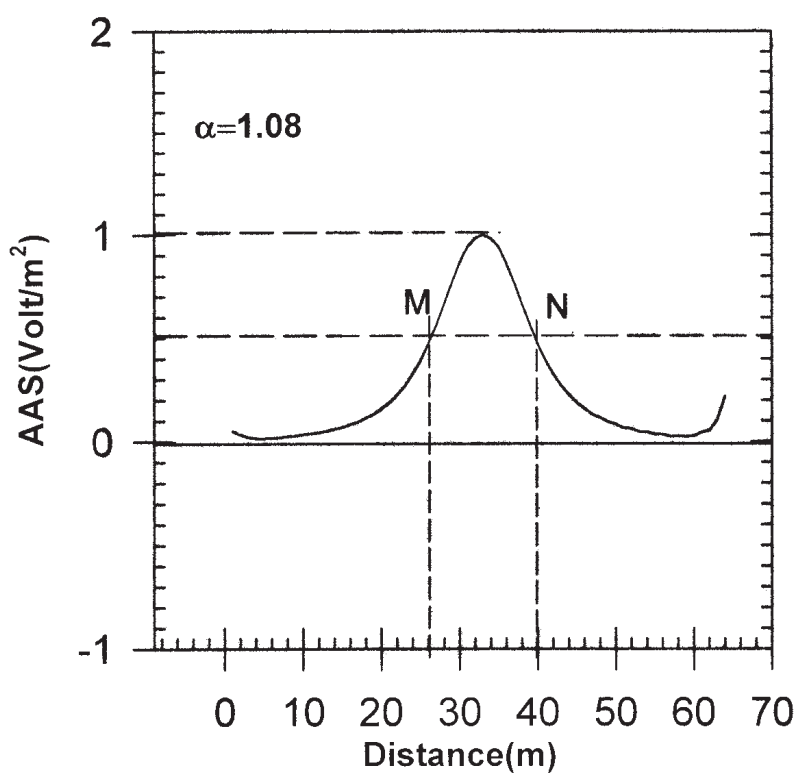

Figure 16. Amplitude of analytic signal (AAS) plot for Model 1.3 and computation of depth to top surface of the body by Nabighian's depth rule. $\mathrm{MN}$ is full width. MN/2 is the estimated depth to top (refer table 3 ). Source is at $S_{0}$. Regularised AAS for Model 1.2 (conductive case). Refer tables 1 and 2 .

The remote current and potential pole positions are also indicated in figure 18(b) for $P_{1}$ current pole (source). For current source pole at $P_{1}$ and $P_{2}$ positions, the current sink, $C_{2}$ is at infinity for all practical purposes $\left(P_{1} C_{1}=11 \mathrm{~m}\right)$. Pole-Pole survey is carried out for three current source positions as indicated in figure 18(a). Henceforth, they are denoted by $P_{1}, P_{2}$ and $P_{3} . P_{1}$ and $P_{2}$ (current pole 


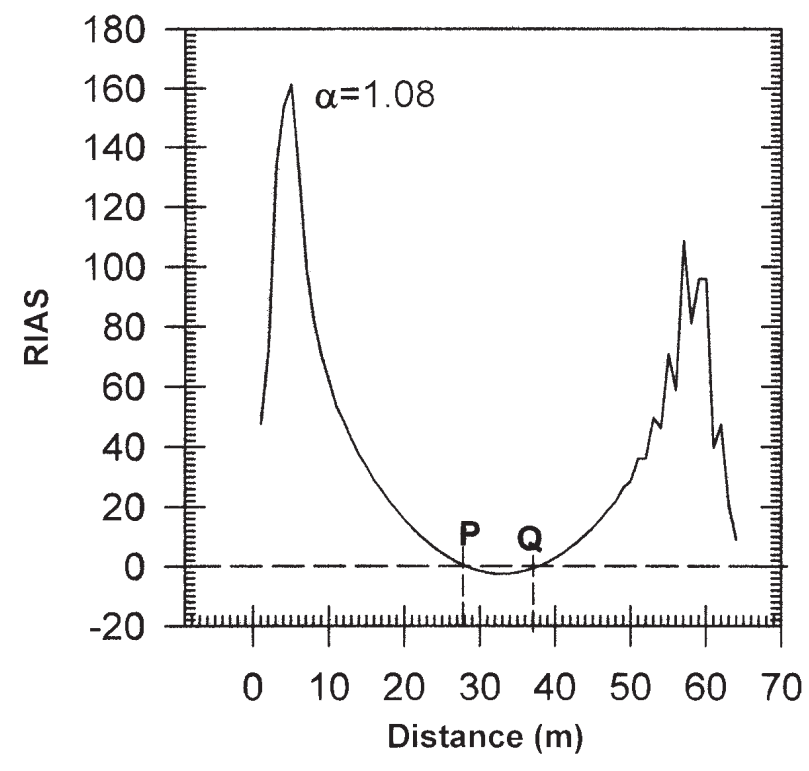

Figure 17. Regularised real amplitude of complex inverse analytic signal (RIAS) plot for Model 1.3 (refer tables 1 and 2 ). Its zero crossings at $P$ and $Q$ identify the lateral edges of the body (refer table 3 ). Source is at $S_{0}$.

Table 4. Geometric details of the iron pipe and physical details of the pipe and host medium.

\begin{tabular}{c|cccc}
\hline \multicolumn{2}{c}{$\begin{array}{c}\text { Diameter } \\
\text { of the } \\
\text { pipe }(\mathrm{m})\end{array}$} & $\begin{array}{c}\text { Depth of } \\
\text { pipe }(\mathrm{m})\end{array}$ & $\begin{array}{c}\text { ConductivityConductivity } \\
\text { of iron } \\
\text { pipe }(\mathrm{S} / \mathrm{m})\end{array}$ & $\begin{array}{c}\text { of host } \\
(\mathrm{S} / \mathrm{m})\end{array}$ \\
\hline Internal & External & 1.9 & $1.0 \mathrm{e}+17$ & 0.17 \\
\hline 0.1524 & 0.2032 & & & \\
\hline
\end{tabular}

positions) are in the vicinity of the pipe, whereas $P_{3}$ is outside its influence. A staggered set of 64 stations is considered for $P_{3} . P_{3}$ is primarily meant for getting the half-space response (in the absence of inhomogeneity).

The pole-pole potential data for $P_{3}$ is subtracted from that of $P_{1}$ and $P_{2}$ current pole positions to yield secondary potential distributions, which are analysed by RES2AS.

The body (iron pipe) position and its lateral extent are attempted for both $P_{1}$ and $P_{2}$ pole positions.

\section{Position location of pipe}

For current pole position $P_{1}$ (situated at $\mathrm{O}$ in figure 18(b)), RAS and IAS plots in figure 19 identify the body location whereas similar plot (figure 20) for an offset current pole position (situated at $2 \mathrm{~N}$ in figure $18(\mathrm{~b})$ ) could not locate the body.

\section{Lateral extent estimation}

The RIAS plot (figure 21) for current source $P_{1}$ provides lateral coordinates and width of pipe. The interpreted results are included in table 5 .

\section{Discussion}

The adopted procedure rests on the analogy between DC electrical and magnetic fields at a fundamental level. However, this analogy may break down for causative sources with open electrical interfaces, primarily because of inhomogeneous electric polarisation set up by external current source(s) as per charge accumulation concept (Li and Oldenburg 1991).

The stabilised analytic signal algorithm, RES2AS uses Tikhonov's regularisation concepts in spectral domain. The adopted regularising operator (equation 12) is equivalent to a dynamic low pass filter, whose properties are determined by smoothing function (equation 13) and regularisation parameter, $\alpha$. Equation (7) outlines an integral equation as an equivalent of nth order derivative for function $u(t)$. This integral equation is the first kind of convolution type and for experimentally measured data, $u(t)$, it belongs to illposed class and equation (8) is an operator form of equation (7).

For error prone input data, the estimated parameters in table 3 are in tune with error amount in conductive model case (Model 1.1).

The adopted pole-pole electrode configuration is a building block for all other in-line electrode configurations and for electrical imaging it is preferred (Li and Oldenburg 1991, 1992). It is hoped that the estimation of secondary potential distribution under field conditions could be more labour intensive for other configurations, but the outlined methodology may still work.

\section{Lateral width determination}

The real part of inverse analytic function (RIAS) has helped in identifying the upper corners of the square shaped targets. The zeroes of RIAS outline the body edges, i.e., the intersection of RIAS curve with horizontal drawn at zero value of RIAS provides the lateral coordinates of the target body. There is no ambiguity and subjective judgement involved in this procedure.

\section{Depth determinations}

Depth estimate is based on Nabighian's depth rule devised for magnetic anomalies. For its applica- 


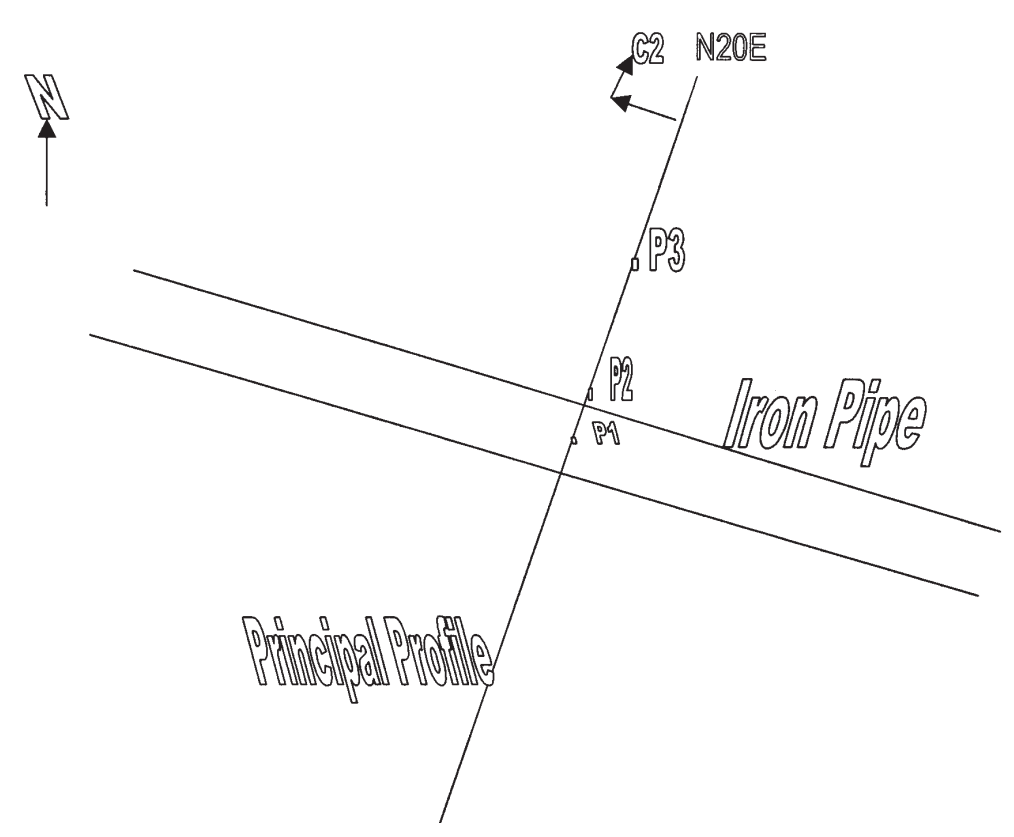

Figure 18(a). Schematic plan view of field layout (pole-pole experiment). $P_{1}, P_{2}$ and $P_{3}$ are current pole positions. Distance between $P_{1}$ and $P_{2}$ is $0.3048 \mathrm{~m}$. Distance between $P_{1}$ and $P_{3}$ is $5.18 \mathrm{~m}$. $C_{2}$ electrode is at a distance of $200 \mathrm{~m}$ (to be at infinity for all practical purposes).

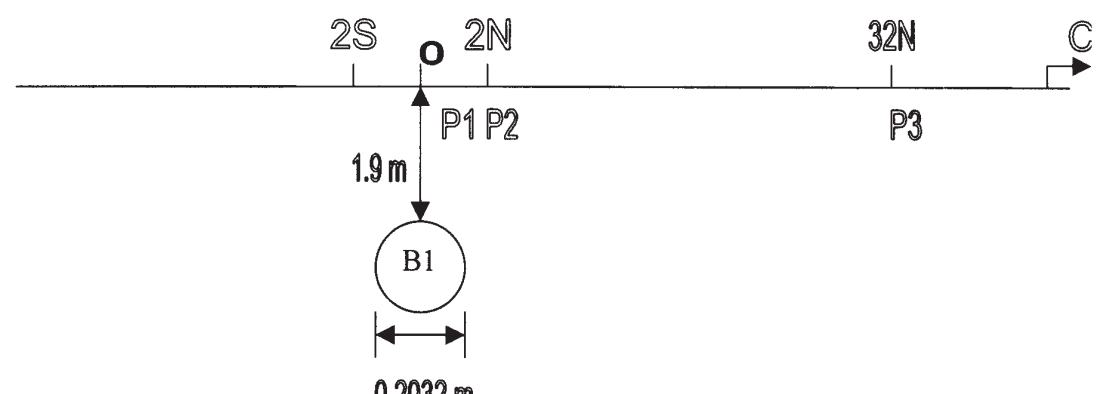

Figure 18(b). Schematic depth section indicating the iron pipe (conductive horizontal cylinder) position and current pole position for pole-pole experiment. Separation between potential electrodes is $0.1524 \mathrm{~m}$.

tion, depth to width ratio has to be within reasonable limits. This depth rule is adopted for our models in view of the similarity in basic governing equations of DC electrical and magnetic methods. Our inability to estimate properly the depth to target body in the case study is because of higher depth to width ratio in excess of unity. There is a need for undertaking rigorous theoretical studies for evolving depth rules in DC resistivity method for targets of closed geometry in accordance with Nabighian's (1976) work. Even though the theory of analytic signal method envisages the depth and lateral coordinates estimation for all body corners, in real practice it may not, due to derivatives in analytic signal emphasising shallow corners at the expense of the deeper ones.

\section{Similarity between secondary pole-pole potential and residual magnetic anomaly}

Geophysical literature is replete with instances of drawing analogies and using them at empirical level. For instance in low-frequency e.m profiling (Telford et al 1976) over closed steeply dipping targets analogy is drawn with vertical and horizontal magnetic anomalies over the same bodies.

\section{Choice of regularisation}

For the various numerical experiments, the AAS and other quantities like RIAS, RAS, IAS are first computed without regularisation $(\alpha=0)$. If the AAS is well behaved (with the maximum amplitude centred), it is considered for further analysis. 


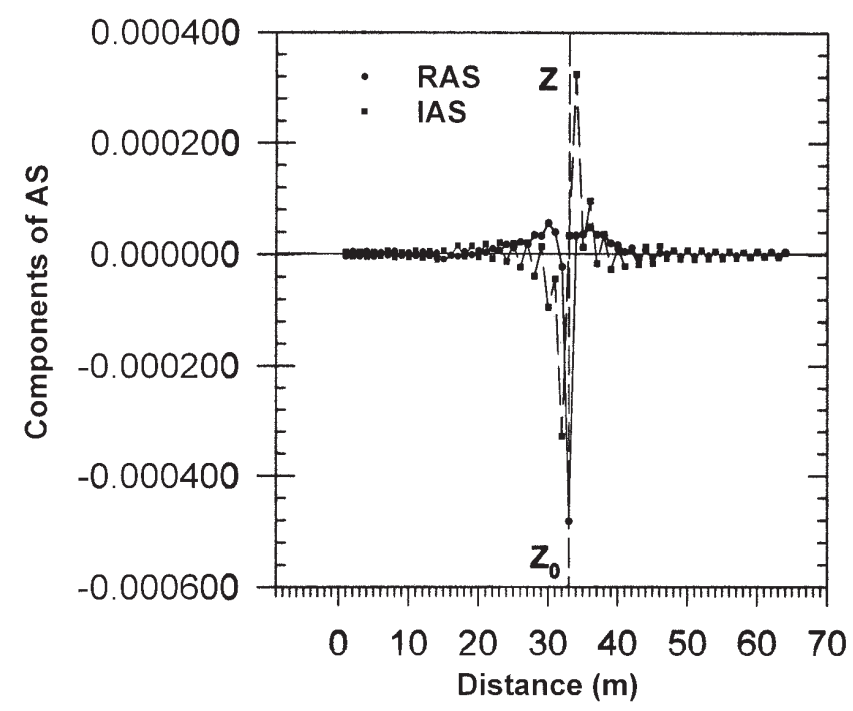

Figure 19. Real and imaginary component plots of analytic signal (RAS and IAS) for source position at $P_{1}$ (refer figure 18). Regularisation is invoked with parameter of regularisation, $\alpha=5.0$. Station interval is $0.1524 \mathrm{~m}$.

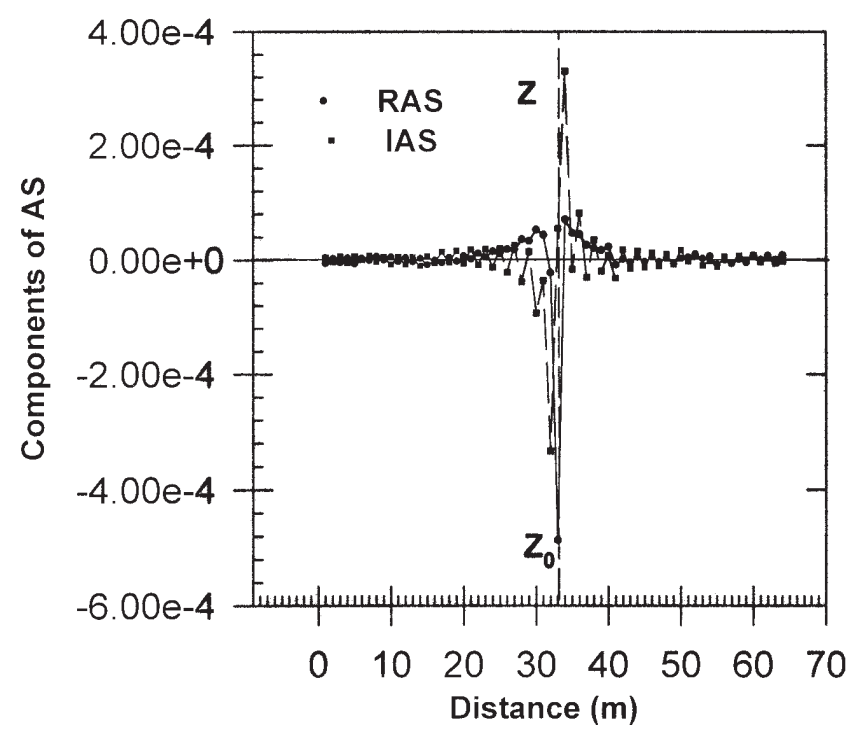

Figure 20. Real and imaginary component plots of analytic signal (RAS and IAS) for source position at $P_{2}$ (refer figure 18). Regularisation is invoked with parameter of regularisation, $\alpha=5.0$. Station interval is $0.1524 \mathrm{~m}$.

Else, regularisation is introduced and regularisation parameter $(\alpha)$ is changed in small increments until AAS behaves properly. The AAS and other quantities like RAS, IAS, and RIAS are then used for interpretation.

The application of this method to other natural electric fields like SP is not tested but it is hoped that it may work as the governing equations are similar.

The robustness of the algorithm is established as evident in figures 7 and 8. This suggests the applicability of the approach in field problems.

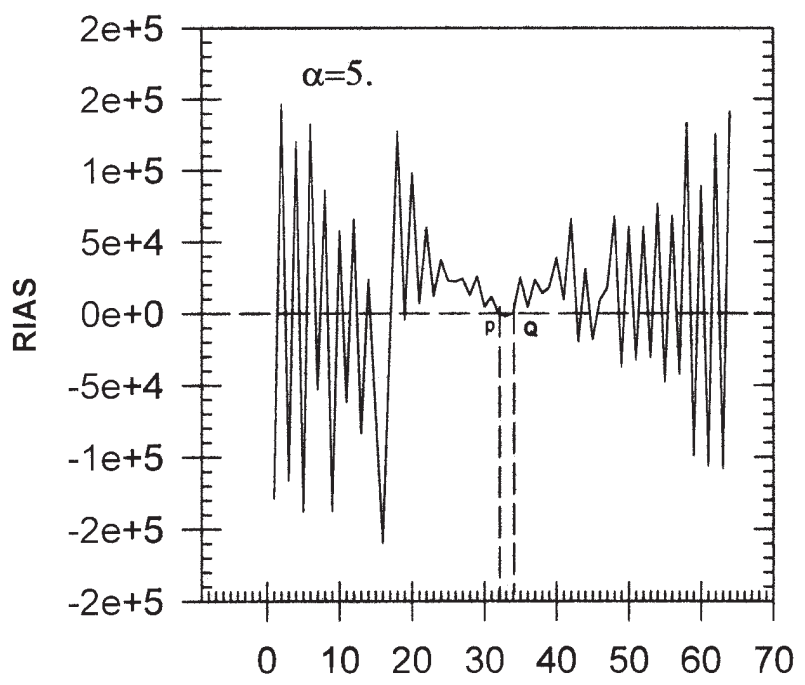

Figure 21. Regularised ( $\alpha=5.0)$ real component of complex inverse analytic signal (RIAS) plot for $(\alpha=5.0)$ for source position $P_{1}$ (refer figure 18). The inferred lateral extent of the target is $P Q$.

Table 5. Interpreted results of field experiment (refer figures 19, 20, 21 and 22).

\begin{tabular}{cccc}
\hline \multicolumn{2}{c}{ Position of the body } & \multicolumn{2}{c}{ Lateral } \\
\hline Actual & Inferred & Actual $(\mathrm{m})$ & Inferred $(\mathrm{m})$ \\
\hline 1S to $1 \mathrm{~N}$ & $1 \mathrm{~S}$ to $1 \mathrm{~N}$ & 0.2032 & 0.2032 \\
\hline
\end{tabular}

\section{Conclusions}

RES2AS has enabled the determination of key body parameters like the depth to top and lateral extent of the body and it worked for both conductive and resistive targets. The study also establishes the stability of the algorithm. The depth estimation is very delicate and it depends on the width to depth ratio in an intricate manner. As the estimated depth is only based on depth rule due to Nabighian (1972), there are discrepancies. However, the lateral depth estimation is more reliable and even for deep seated bodies, use of a strong regularisation has enabled determination of the body edges fairly well.

RES2AS requires a total CPU time of 39.1 seconds to compute the pole-pole potentials and analytic signal and its associated terms for a typical $51 \times 101$ grid size. Out of which, 37.2 seconds are needed for computation of the forward response (secondary potential) only. These computations are made on DEC ALPHA 400 series (64-bit machine) with $166 \mathrm{MHz}$ processor and $64 \mathrm{Mb}$ RAM. The CPU time on a PC 486 did not exceed 1 minute for computation of Stabilised Analytic Signal and its associated terms with previous input. 


\section{Acknowledgements}

The authors are grateful to the Head, Department of Earth Sciences for providing necessary facilities. One of the authors (PR Pujari) acknowledges the financial support received from C.S.I.R., India.

\section{List of symbols}

$\begin{array}{ll}\alpha & \text { Regularising parameter. } \\ \sigma^{*} & \text { Volume density of dipoles. } \\ \mu_{i}(i=1,2) & \begin{array}{l}\text { Magnetic permeability of } i^{\text {th }} \\ \text { medium. }\end{array} \\ \rho_{i}(i=1,2) & \text { True resistivity of } i^{\text {th }} \text { medium. } \\ V_{i}(i=1,2) & \begin{array}{l}\text { DC pole-pole electric potential in } \\ \text { ith medium. }\end{array} \\ V_{s} & \begin{array}{l}\text { Secondary pole-pole potential in } \\ \text { the first medium. }\end{array} \\ u(t) & \text { Arbitrary input function. } \\ z(t) & \text { First derivative of } u(t) . \\ A(x, z) & \text { Analytic signal }(\mathrm{AS}) \text { of secondary } \\ & \text { DC pole-pole potential, } u_{s} . \\ f(\omega, \alpha) & \text { Order of regularization. } \\ & \text { Tikhonov's regularizing operator. }\end{array}$

\section{References}

Eskola L 1992 Geophysical interpretation using integral equations; (London: Chapman and Hall) 191p

Li Y and Oldenburg D W 1991 Aspects of charge accumulation in DC resistivity experiments; Geophysical Prospecting 39 803-826
Li Y and Oldenburg D W 1992 Approximate inverse mappings in DC resistivity problems; Geophys. J. Int. 109 343-362

Mufti I R 1976 Finite difference resistivity modelling for arbitrarily shaped two dimensional structures; Geophysics $\mathbf{4 1}$ 62-78

Nabighian M N 1972 The analytic signal of two-dimensional magnetic bodies with polygonal cross-section; Geophysics 37 507-517

Nabighian M N 1984 Towards a three-dimensional automatic interpretation of potential field data via generalized Hilbert Transforms: Fundamental relations; Geophysics 49 780-786

Pujari P R 1998 Stabilised analytic signal algorithm for 2D/3D DC resistivity data analysis; Ph.D. thesis, University of Roorkee

Quick D H 1974 The interpretation of gradient array chargeability anomalies; Geophysical Prospecting 22 736-746

Rao Atchuta D, Ram Babu H V and Shankar Narayan P V 1981 The complex gradient method; Geophysics 6 15721578

Roest W R, Verhoef J and Pilkington M 1992 Magnetic interpretation using 3-D analytic signal; Geophysics $\mathbf{5 7}$ $116-125$

Starostenko V I and Sastry R G S 1979 Regulariziyusheye resheniye trayohmerniih zadatch geofiziki pridctavlennih integralnimii uravneniyami pervova roda tipa sveortiki; Dokladii Akademii Nauk SSSR 2465 1074-1079

Sunderrajan N and Srinivas Y 1996 A modified Hilbert transform and its application to self potential interpretation; Applied Geophysics 36 137-143

Sunderrajan N, Srinivasa Rao P and Sunitha V 1998 An analytical method to interpret self-potential anomalies caused by 2-D inclined sheets; Geophysics 63 1551-1555

Telford W M, Geldart L P, Sheriff R E and Keys D A 1976 Applied Geophysics (Cambridge University Press) 860 pp.

Tikhonov A N and Arsenin V 1977 Solution of ill-posed problems (Washington: John Wiley) 